\section{ORIGINAL RESEARCH}

L. Zhang

Q. Huang

Y. Zhang

J. Liu

B. Hong

Y. Xu

W. Zhao

\title{
Wingspan Stents for the Treatment of Symptomatic Atherosclerotic Stenosis in Small Intracranial Vessels: Safety and Efficacy Evaluation
}

BACKGROUND AND PURPOSE: Until now, endovascular treatment of symptomatic atherosclerotic stenosis in small intracranial arteries $(\leq 2.5 \mathrm{~mm})$ was limited. We evaluated the safety and efficacy of the treatment by using Wingspan stents in arteries of this caliber.

MATERIALS AND METHODS: From March 2007 to July 2010, 53 symptomatic intracranial stenoses with narrowing of at least $50 \%$ in 53 patients were treated by using Wingspan stents. Clinical manifestations and imaging features were recorded.

RESULTS: The technical success rate was $98.1 \%$. There were no serious complications, with the exception of 1 patient who experienced a small cerebral hemorrhage caused by perforation of microwire. Thirty-nine patients (74\%) were available for follow-up imaging with DSA. ISR was documented in 13 of these patients, including 2 patients with symptomatic ISR. The median length of the vascular lesions was $5.39 \mathrm{~mm}$, and patients whose vascular lesions were longer than $5.39 \mathrm{~mm}$ had a much higher incidence of ISR than patients whose vascular lesions were shorter than $5.39 \mathrm{~mm}(53 \%$ versus $15 \%$, respectively). The median ratio of the reference artery diameter to the stent diameter was 0.78 , and patients whose ratio was smaller than 0.78 had a much higher incidence of ISR than patients whose ratio was larger than 0.78 (53\% versus $15 \%$, respectively).

CoNCLUSIONS: In our series, percutaneous transluminal angioplasty and stent placement of small intracranial arteries by using Wingspan stents was safe. The ISR rate was relatively high; most patients having ISR were asymptomatic. Further follow-up is needed to assess the long-term efficacy of this procedure.

ABBREVIATIONS: $A C T=$ activated clotting time; ISR = in-stent restenosis; LMA = location, morphology, and access; PTAS = percutaneous transluminal angioplasty and stenting; WASID = Warfarin Aspirin Symptomatic Intracranial Disease

$\mathbf{P}$ TAS is an important method for treating atherosclerotic stenosis in intracranial arteries, and preliminary results suggest that this procedure is safe and effective. PTAS is difficult to implement in small-diameter artery stenosis because of the high surgical skill required and the high rate of restenosis. ${ }^{1,2}$ From experience we know that the incidence of ISR is $25 \%$ with angioplasty and stent placement in small coronary arteries. ${ }^{3}$ Vessel diameter is negatively correlated with ISR and other adverse outcomes. ${ }^{4}$

By using the self-expanding Wingspan stent system (Boston Scientific, Freemont, California) to treat intracranial artery stenosis, the surgical success rate and safety of the procedure are further improved because of increased compliance. Bose et $\mathrm{al}^{5}$ studied patients with intracranial stenosis in arter-

Received March 15, 2011; accepted after revision June 8

From the Department of Neurosurgery, Changhai Hospital, Second Military Medical University, Shanghai, China.

L.Z. and $\mathbf{Q} . \mathrm{H}$. contributed equally to this paper.

This work was supported in part by the Key Project from the Shanghai Committee of Science and Technology (074119506).

Please address correspondence to Jianming Liu, Neurosurgery of Changhai Hospital, Second Military Medical University, 168 Changhai Rd, Shanghai 200433, China; e-mail: liu118@vip.163.com

Indicates open access to non-subscribers at www.ajnr.org

ies 2.5 to $4.5 \mathrm{~mm}$ in diameter and who were treated with a Wingspan stent; however, to date, only a few studies have been conducted on the use of Wingspan stents in the treatment of small intracranial artery stenosis, and the sample size was small. ${ }^{6,7}$ Here, we defined small intracranial arteries as those with a vessel diameter $\leq 2.5 \mathrm{~mm}$. In this study of 53 patients, with 53 small intracranial stenoses that were treated with Wingspan stents, we used clinical manifestations and imaging results to assess the safety and follow-up results of the procedure.

\section{Materials and Methods}

\section{Patients and Techniques}

We reviewed patients with symptomatic atherosclerotic stenosis in small intracranial arteries $(\leq 2.5 \mathrm{~mm}$ ) treated with Wingspan stents in our hospital during a 41-month period from March 2007 to July 2010. We carefully reviewed the detailed patient information, including the patient's age and sex, clinical manifestations, lesion morphology, and endovascular treatment strategy.

Inclusion criteria were as follows: 1) age between 18 and 75 years; 2) at minimum, a TIA or stroke related to the symptomatic atherosclerotic stenosis in an intracranial artery within the preceding 180 days; 3 ) a pre-event $\mathrm{mRS}$ score $\leq 3$; and 4 ) a DSA showing reference artery diameter $\leq 2.5 \mathrm{~mm}$.

Exclusion criteria were as follows: 1) nonatherosclerotic intracra- 
nial arterial stenosis; 2) patients with brain tumors, vascular malformations, or aneurysms; 3) cardiogenic stroke in patients with atrial fibrillation, heart valve disease, left ventricular mural thrombus, or left ventricular myocardial infarction within the preceding 6 weeks; 4) patients with contraindications to the contrast agent, heparin, or anesthesia; and 5) having the same lesion previously treated with a stent.

\section{Intervention Procedure}

In brief, access was typically achieved through the common femoral artery. Heparin was titrated during the procedure to achieve an ACT that was 2 to 2.5 times that of baseline. Almost all procedures were performed through a $6 \mathrm{~F}$ guiding catheter or a long-sheath system. After conventional catheter-based angiography, a microcatheter was manipulated across the target lesion by using a 0.014-inch microwire (Transend EX Platinum, Boston Scientific; ATW, Cordis Corp., Miami, Florida). The microcatheter was then exchanged over a 0.014 inch exchange microwire for a Gateway angioplasty balloon (Boston Scientific). When the reference artery diameter was between $1.85 \mathrm{~mm}$ and $2.5 \mathrm{~mm}$ (including $1.85 \mathrm{~mm}$ and $2.5 \mathrm{~mm}$ ), the balloon diameter used was $80 \%$ of the reference artery diameter. When the reference artery diameter was between $1.5 \mathrm{~mm}$ and $1.85 \mathrm{~mm}$ (including 1.5 $\mathrm{mm}$ ), we chose a balloon with a diameter of $1.5 \mathrm{~mm}$. When the reference artery diameter was below $1.5 \mathrm{~mm}$, we chose a balloon with a diameter of $1.5 \mathrm{~mm}$ ( 1 patient's artery had a diameter of $1.44 \mathrm{~mm}$ ). The balloon length was selected to match the length of the lesion. Angioplasty was typically performed with a slow, graded inflation of the balloon to a pressure of between 6 and $10 \mathrm{~atm}$, and was then maintained for 15-20 seconds. Following angioplasty, the balloon was removed and conventional angiography was repeated. Next, the Wingspan delivery system was prepared and advanced over the exchange wire across the target lesion. The stent's diameter was chosen to exceed the diameter of the reference artery by $0.5-1.0 \mathrm{~mm}$. The stent's length was selected to exceed the length of the lesion by least 3 $\mathrm{mm}$ on both sides. Angiography was performed to measure residual postoperative stenosis.

The procedures were performed by neurointervention physicians, (Q.H., J.L., B.H., Y.X., W.Z.). All have more than 8 years of experience in neurointervention.

\section{Preprocedure and Postprocedure Medical Therapy}

All patients were pretreated with a daily dose of $75 \mathrm{mg}$ clopidogrel and $300 \mathrm{mg}$ aspirin at least 3 days before the endovascular procedure. Aspirin was maintained at a daily dose of $300 \mathrm{mg}$ for at least 6 months after the procedure until follow-up angiography was performed. In cases where no ISR or other related disease developed, aspirin was usually continued indefinitely at a daily dose of $100 \mathrm{mg}$. Clopidogrel was usually maintained for 6 weeks after surgery and then discontinued. Risk factors for atherosclerosis were controlled in accordance with relevant postprocedural guidelines.

\section{Clinical and Angiographic Follow-Up}

Clinical scores (modified Rankin score and NIHSS score) were obtained before the procedure, after the procedure, and before discharge. Following surgery, scores were obtained at 1 day, 4 weeks, 6 months, 1 year, and every other year thereafter. Follow-up angiography was performed at 6 months. ISR was defined as $>50 \%$ stenosis within or immediately adjacent to (within $5 \mathrm{~mm}$ ) the implanted stent, with $>20 \%$ absolute luminal loss. ${ }^{8}$ The initial and follow-up clinical examinations were performed by 1 neurologist (Y.Z.).

\section{Statistical Methods}

Before the measurements were taken, the measuring method was clearly identified by the investigators; the measurements were taken by 2 experienced investigators. Despite this, measurement bias is always inevitable, so we performed a $t$ test for the 2 sets of data and found no statistically significant difference $(P>.05)$. We therefore took the average of the 2 sets of data as the final measurement.

Discrete lesions treated in the same vascular distribution were counted as a single patient with 2 treated lesions, and the stents were evaluated independently for ISR. If lesions were treated in 2 separate vascular distributions in 1 patient, then that patient was counted twice, with each stent evaluated independently for ISR. We grouped the patients according to the following criteria: relationship between ISR and lesion site, degree of vascular tortuosity, length of the vascular lesion, reference artery diameter and ratio of the reference artery diameter to the stent diameter, and residual stenosis. The count data were then analyzed by using the $\chi$-square test. A $P$ value of $<0.05$ represented a statistically significant difference. The data were analyzed by using SPSS 16.0 software.

\section{Results}

\section{Patient Characteristics}

The study included 53 patients ( 40 men and 13 women) ranging in age from 41 to 75 years (mean age, 56.7 years). Before stent placement, a total of 25 patients (47\%) had experienced TIAs and 28 patients (53\%) had experienced a stroke. Median time from qualifying event to stent placement ranged from 1 day to 45 days, with a mean of 23 days.

All 53 patients received DSA preoperatively. We defined the distal artery diameter as the reference artery diameter. The degree or percent stenosis of the target lesion was determined by using the formulas described by the WASID method. ${ }^{9}$ Of the 53 lesions, 45 (84.9\%) were located in an anterior circulation and were distributed as follows: 2 were in the intracranial carotid artery, 40 were in the M1 segment of the middle cerebral artery, and 3 were in the M2 segment of the middle cerebral artery; the remaining 8 lesions $(15.1 \%)$ were in the posterior circulation. Artery diameter ranged from 1.44 to $2.5 \mathrm{~mm}$, with a mean of $2.07 \mathrm{~mm}$. All vascular lesions had 50\%-95\% narrowing, with a mean of $73.9 \pm 2.7 \%$. In total, 42 of the 53 (79.2\%) stenoses were greater than $70 \%$.

\section{Treatment Results}

Initial Treatment Results. To date, we attempted implantation of 54 Wingspan stents in 53 patients. The length of stenosis in 1 patient was $28.4 \mathrm{~mm}$, so we needed 2 stents to cover the lesion. The technical success rate for stent deployment across the stenotic lesion was 98.1\% (Fig 1); only 1 stent could not be released at the target site because of vascular tortuosity. All of the patients were treated with a Gateway balloon and a Wingspan stent. After Gateway angioplasty, the stenoses were $10 \%-80 \%$ (with a mean of $40.9 \pm 4.2 \%$ ). After Wingspan stent placement, the residual stenoses were $0 \%$ $50 \%$ (with a mean of $13.0 \pm 3.4 \%$ ). One patient (1.9\%) experienced surgery-related complications during the perioperative period. This patient, with middle cerebral artery stenosis, accepted an implanted Wingspan stent. The CT after the procedure showed a small hematoma in the basal ganglia region, and the hematoma did not increase. This may have been 

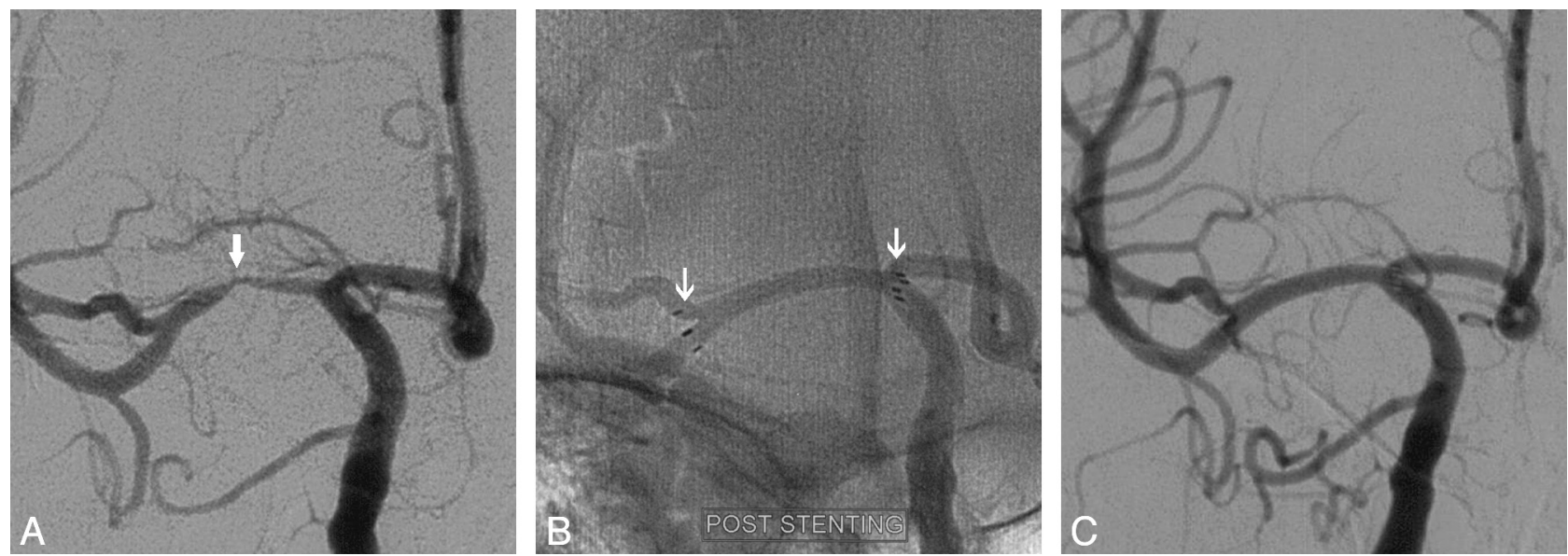

Fig 1. A 42-year-old man with a severe stenosis of the right middle cerebral artery (MCA) was referred for evaluation of TIAs recurrent. Diagnostic cerebral angiography confirmed a preocclusive $(>75 \%)$ stenosis of the right MCA $(A$, arrow). The patient underwent percutaneous transluminal angioplasty and stent placement with Wingspan. The subtracted image demonstrates near-complete resolution of the stenosis $(B$, arrow). The patient's ischemic symptoms immediately resolved. The 6-month follow-up angiogram shows continued patency of the stented segment $(C)$.
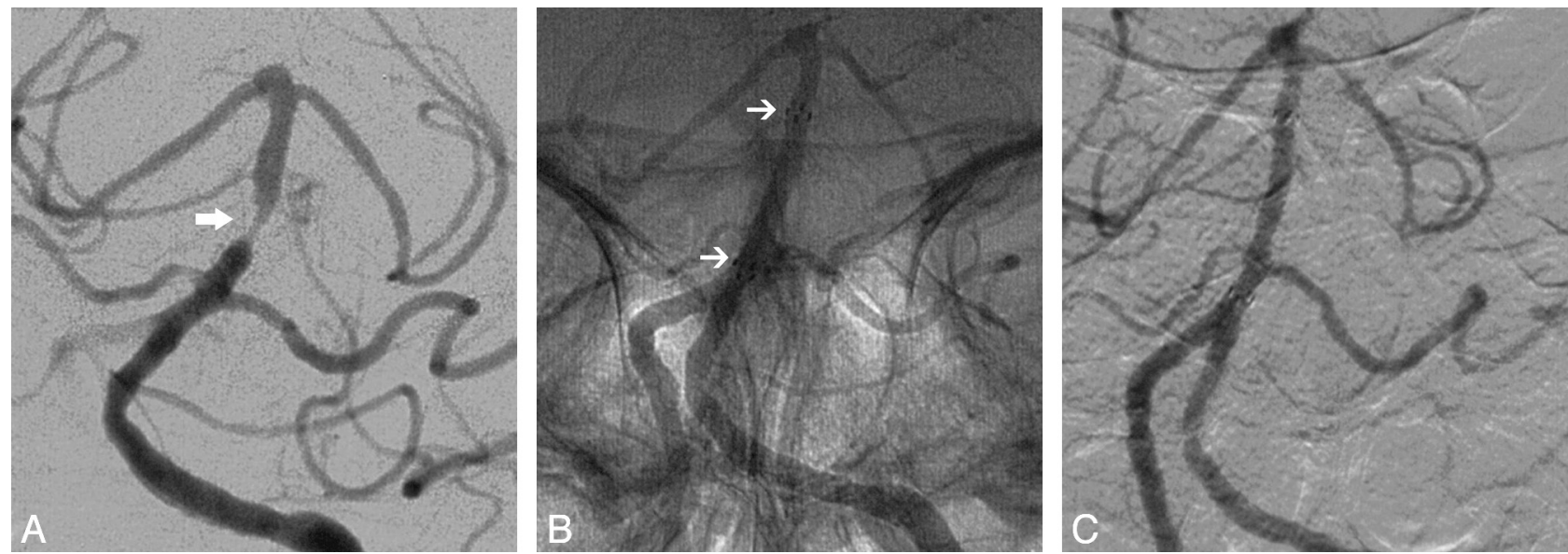

Fig 2. A 60-year-old woman underwent percutaneous transluminal angioplasty and stent placement with Wingspan for a symptomatic basal artery stenosis with TIAs despite taking aspirin and clopidogrel $(A$ and $B$, arrows). The patient's ischemic symptoms completely resolved after the procedure. The 17-month follow-up angiogram demonstrates there was no ISR $(C)$.

caused by a perforation from the microwire. The patient suffered partial anomic aphasia for 1 day and mild muscle weakness for 2 weeks, and then gradually returned to normal. This perforation did not lead to permanent neurologic sequelae. No patients died during the perioperative period.

Imaging and Clinical Follow-Up. Among 52 patients successfully treated by Wingspan stents, 39 patients (74\%) were available for follow-up imaging with DSA (Fig 2). The imaging follow-up time was 6-32 months, with a mean of 9.8 months. ISR was documented in 13 patients $(33.3 \%)$, including 1 patient with complete stent occlusion. One ISR patient received angioplasty with a drug-eluting stent. Fifty patients were available for a clinical follow-up after 6-40 months, with a mean of 17.9 months; 34 patients had their follow-up more than 12 months after surgery. Of the 50 patients, 4 had clinical symptoms: 2 had symptomatic ISR and 2 had TIAs in a nonstented region. No patients died during the follow-up period.

\section{Risk Factors for ISR}

We calculated the median length of the vascular lesions (5.39 $\mathrm{mm}$ ), the median ratio of the reference artery diameter to the
Relationship between ISR and clinical or anatomic characteristics

\begin{tabular}{lccc}
\hline & ISR & Non-ISR & $P$ \\
\hline Lesion site & & & \\
$\quad$ Anterior $(n=31)$ & 8 & 23 & NS \\
$\quad$ Posterior $(n=8)$ & 5 & 3 & \\
Degree of vascular tortuosity & & & \\
$\quad$ Tortuous $(n=18)$ & 6 & 12 & NS \\
$\quad$ Direct $(n=21)$ & 7 & 14 & \\
Reference artery diameter/stent diameter & & & \\
$\quad<0.78(n=19)$ & 10 & 9 & 0.013 \\
$\quad \geq 0.78(n=20)$ & 3 & 17 & \\
Length of vascular lesions & & & \\
$\quad<5.39 \mathrm{~mm}(n=20)$ & 3 & 17 & 0.013 \\
$\quad \geq 5.39 \mathrm{~mm}(n=19)$ & 10 & 9 & \\
Residual stenosis & & & \\
$\quad>10.7 \%(n=24)$ & 7 & 17 & NS \\
$\quad \leq 10.7 \%(n=15)$ & 6 & 9 & \\
Reference artery diameter & & & \\
$\quad \leq 2.5 \mathrm{~mm}$ and $>2 \mathrm{~mm}(n=24)$ & 7 & 17 & \\
$\quad \leq 2 \mathrm{~mm}$ and $>1.5 \mathrm{~mm}(n=13)$ & 5 & 8 & NS \\
$\quad \leq 1.5 \mathrm{~mm}(n=2)$ & 1 & 1 & \\
\hline
\end{tabular}

Note:- The relationship between ISR and each independent factor was analyzed by the Pearson $\chi$-square test. NS indicates nonsignificant. 
stent diameter (0.78), and the average degree of residual stenosis $(10.7 \%)$ among patients who had a follow-up, then grouped the patients based on whether they fell above or below these mean values (Table). The results showed that patients with vascular lesions longer than $5.39 \mathrm{~mm}$ and/or ratios smaller than 0.78 had a much higher incidence of ISR.

\section{Discussion}

Although PTAS is an important method for treating cerebral artery stenosis, PTAS for the treatment of small intracranial artery stenosis remains challenging. Based on experience in the treatment of coronary stenosis, vessel size is inversely correlated with the risk of restenosis and with adverse outcomes after percutaneous coronary intervention ${ }^{4}$; this is because a small vessel is less able to accommodate lumen renarrowing, which invariably occurs, to some degree, in most arteries after balloon dilation. ${ }^{10}$ Moreover, intracranial arteries are more fragile than peripheral arteries due to their thin muscle in the artery wall, their lack of support by peripheral tissues, and their vascular tortuosity and small vessel diameter; these features make stent-assisted angioplasty procedures more difficult and possibly increase the incidence of complications. Thus, intracranial PTAS, especially in small intracranial arteries, is challenging.

Wingspan stents have made it possible to use PTAS to treat small intracranial arteries. In our study, the technical success rate was $98.1 \%$; 1 stent could not be released to the target site because of vascular tortuosity. Although there was 1 surgeryrelated complication during the perioperative period, this patient had no permanent neurologic sequelae. Before placement of the Wingspan stent, an angioplasty was performed using a Gateway balloon. This conservative predilation approach reduces the degree of vascular trauma and likely minimizes both the risk of target-vessel perforation and the likelihood of downstream embolization of atheromatous debris caused by plaque disruption. Several research studies reported that the incidence of complications was 15\%-30\% when using a coronary balloon stent for intracranial stenosis, whereas the incidence was about 5\%-18\% when using a Gateway balloon and Wingspan stent. ${ }^{10-19}$ After Wingspan stent placement, the residual stenoses were $0 \%-50 \%$ (with a mean of $13.0 \pm 3.4 \%)$. The average residual stenoses of patients who had a follow-up was $10.7 \%$. It was better than the $23 \%-36 \%$ reported, maybe related to the small caliber. First, there were 15 patients with reference artery diameter $<1.85 \mathrm{~mm}$; we chose a balloon with a diameter larger than $80 \%$ of the reference artery diameter. Second, angioplasty was typically performed with a slow-graded inflation of the balloon to a pressure higher than nominal pressure. Third, we usually chose a stent with larger diameter to have good dilation. So the degree of predilation with the balloon was greater.

ISR is a key factor influencing the outcome of PTAS. The rate of ISR in patients who undergo coronary vessel PTAS with a bare metal stent can be as high as $27.8 \% .{ }^{19}$ The occurrence of ISR is related to vascular injury and excessive repair. In theory, Wingspan stents can prevent ISR by reducing vascular injury, but the incidence of ISR remains at $30 \% .^{20,21}$ ISR is more common in small coronary arteries, ${ }^{4}$ but there are no related reports for small intracranial artery stenosis. In our study, among the 39 patients who received an imaging follow-up, we encountered 13 patients (33.3\%) with ISR. This percentage is similar to the currently reported incidence of ISR. Meanwhile, only 2 out of $13(15.4 \%)$ patients were symptomatic. Levy et $\mathrm{al}^{22}$ showed that $24 \%$ of patients with ISR were symptomatic, and some single-center studies obtained similar results. ${ }^{8,23}$ These data suggest that most patients with ISR are asymptomatic.

The lesion site, length of the vascular lesion, residual stenosis, degree of vascular tortuosity, reference artery diameter, and stent diameter are all potential risk factors for ISR. Fiorella et $\mathrm{al}^{24}$ found that anterior circulation lesions were much more prone to ISR than posterior circulation lesions. But they did not reveal any underlying characteristics that exposed patients to an increased risk of ISR, with the exception of lesion location. ${ }^{22}$ In our research, the differences were not statistically significant because of the small sample size. Turk et $\mathrm{al}^{25}$ showed that anterior circulation regions-in particular, the supraclinoid segment and the middle cerebral artery-were highly prone to restenosis, and ISR lesions in these 2 locations were often more serious than the original stenosis. ${ }^{20}$ It may be related to the degree of vascular tortuosity. In our dataset, we divided patients into 2 groups, according to the LMA classification, but found no statistically significant differences. On the one hand, vascular tortuosity increases surgical difficulty, and the stent cannot be released to the target site; on the other hand, because of malapposition, the stent cannot effectively support the wall or control the progress of the atherosclerotic plaque. Both of these situations can increase the incidence of ISR. In the future, it will be necessary to study the stent morphology after release by using CT angiography flat panel angioCT (Dyn-CT).

Some researchers also studied the relationship between the length of the vascular lesion or residual stenosis and ISR. They showed that patients with long lesions and a high percentage of residual stenosis have high rates of restenosis. ${ }^{26,27}$ In our study, we divided 39 patients with imaging follow-up into 2 groups based on the median length of the vascular lesions $(5.39 \mathrm{~mm})$. Patients whose vascular lesions were longer than $5.39 \mathrm{~mm}$ had a much higher incidence of ISR (53\% versus $15 \%$ for patients with a lesion length of $5.39 \mathrm{~mm} ; P<.05)$. The median degree of residual stenosis was $10.7 \%$, and we used the same method to group the patients but found no statistically significant difference between the 2 groups.

The self-expanding Wingspan stent exerts a continuous outward radial force against the vessel wall. This outward radial force prevents early vessel recoil, thus consolidating the gains achieved with the initial angioplasty. A recent study showed that the degree of undersizing significantly affected wall shear stress, the wall shear stress gradient, and the oscillatory shear index, which may promote intimal hyperplasia, thrombosis, and atherogenesis. Conversely, oversizing significantly increased intramural stress, which can cause acute vessel dissection and can chronically stimulate smooth muscle proliferation and initiate an inflammatory response. ${ }^{28}$ Thus, both undersizing and oversizing can lead to ISR. In the course of treatment detailed in our study, the Wingspan stent was slightly oversized, measuring 0.5 to $1.0 \mathrm{~mm}$ larger than the diameter of the reference artery. Based on the median ratio of the reference artery diameter to the stent diameter (0.78), we divided the 39 patients who received follow-up imaging into 2 
groups. Patients whose ratio was less than 0.78 had a much higher incidence of ISR (53\% versus $15 \%$ for patients with a ratio of more than $0.78 ; P<.05)$. It is possible that the intramural wall stress from the stent caused more damage to the vessel wall and subsequent excessive repair, ultimately leading to ISR. ${ }^{26}$ Therefore, choosing a stent of the appropriate size may effectively reduce the blood flow dynamics and mechanical interference on the vessel wall, thereby reducing the incidence of ISR.

The limitations of these data should be noted. First, angiographic follow-up of all patients has not yet been completed, and the absolute number of follow-up patients becomes relatively small when they are split into subsets for analysis. Second, this is a restrospective analysis, so selection bias may be built into the data. Prospective randomized controlled trials are needed to provide more adequate statistical evidence. Third, there is insufficient relevant data on the use of Wingspan stents to assess the long-term efficacy of this procedure.

\section{Conclusions}

In our series, percutaneous transluminal angioplasty and stent placement of small intracranial arteries was safe. The ISR rate was relatively high; most patients having ISR were asymptomatic. Patients with longer vascular lesions and smaller ratios of the reference artery diameter to the stent diameter were more prone to ISR. Further follow-up is needed to assess the longterm efficacy of this procedure.

\section{References}

1. Moreno R, Fernández C, Alfonso F, et al. Coronary stenting versus balloon angioplasty in small vessels: a meta-analysis from 11 randomized studies. J Am Coll Cardiol 2004;43:1964-72

2. Costa MA, Sabate M, Staico R, et al. Anatomical and physiologic assessments in patients with small coronary artery disease: final results of the Physiologic and Anatomical Evaluation Prior to and After Stent Implantation in Small Coronary Vessels (PHANTOM) trial. Am Heart J 2007;153:296.e1-7

3. Moussavian M, Casterella PJ, Teirstein PS. Restenosis after angioplasty. Curr Treat Options Cardiovasc Med 2001;3:103-13

4. Elezi S, Kastrati A, Neumann FJ, et al. Vessel size and long-term outcome after coronary stent placement. Circulation 1998;98:1875-80

5. Bose A, Hartmann M, Henkes $\mathrm{H}$, et al. A novel, self-expanding, nitinol stent in medically refractory intracranial atherosclerotic stenoses: the Wingspan study. Stroke 2007;38:1531-37

6. Turk AS, Ahmed A, Niemann DB, et al. Utilization of self-expanding stents in the treatment of intracranial atherosclerotic disease in the distal small cerebral vessels. Neuroradiology 2007;49:659-63

7. Turk AS, Niemann DB, Ahmed A, et al. Use of self-expanding stents in distal small cerebral vessels. AJNR Am J Neuroradiol 2007;28:533-36
8. Kim DJ, Lee BH, Kim DI, et al. Stent-assisted angioplasty of symptomatic intracranial vertebrobasilar artery stenosis: feasibility and follow-up results. AJNR Am J Neuroradiol 2005;16:1381-88

9. Samuels OB, Joseph GL, Lynn MJ, et al. A standardized method for measuring intracranial arterial stenosis. AJNR Am J Neuroradiol 2000;21:643-46

10. Li JJ, Xu B, Yang YJ, et al. Drug-eluting stent for the treatment of small coronary lesion: comparison between sirolimus- and paclitaxel-eluting stent. Chin Med J 2007;120:569-73

11. Chow MM, Masaryk TJ, Woo HH, et al. Stent-assisted angioplasty of intracranial vertebrobasilar atherosclerosis: midterm analysis of clinical and radiologic predictors of neurological morbidity and mortality. AJNR Am J Neuroradiol 2005;26:869-74

12. Kim JK, Ahn JY, Lee BH, et al. Elective stenting for symptomatic middle cerebral artery stenosis presenting as transient ischaemic deficits or stroke attacks: short term arteriographical and clinical outcome. J Neurol Neurosurg Psychiatry 2004;75:847-51

13. Lee TH, Kim DH, Lee BH, et al. Preliminary results of endovascular stentassisted angioplasty for symptomatic middle cerebral artery stenosis. AJNR Am J Neuroradiol 2005;26:166-74

14. Weber W, Mayer TE, Henkes $H$, et al. Stent-angioplasty of intracranial vertebral and basilar artery stenoses in symptomatic patients. Eur J Radiol 2005;55:231-36

15. Fiorella D, Levy EI, Turk AI, et al. US multicenter experience with the Wingspan stent system for the treatment of intracranial atheromatous disease: periprocedural results. Stroke 2007;38:881-87

16. Zaidat OO, Klucznik R, Alexander MJ, et al. NIH multi-center Wingspan Intracranial Stent Registry Study Group. The NIH registry on use of the Wingspan stent for symptomatic 70-99\% intracranial arterial stenosis. Neurology 2008;70:1518-24

17. Zhao ZW, Deng JP, He SM, et al. Intracranial angioplasty with Gateway-Wingspan system for symptomatic atherosclerotic stenosis: preliminary results of 27 Chinese patients. Surg Neurol 2009;72:607-11

18. Lanfranconi S, Bersano A, Branca V, et al. Stenting for the treatment of highgrade intracranial stenoses. J Neurol 2010;257:1899-908

19. Agostoni P, Biondi-Zoccai GG, Gasparini GL, et al. Is bare-metal stenting superior to balloon angioplasty for small vessel coronary artery disease? Evidence from a meta-analysis of randomized trials. Eur Heart J 2005;26:881-89

20. Albuquerque FC, Levy EI, Turk AS, et al. Angiographic patterns of Wingspan in-stent restenosis. Neurosurgery 2008;63:23-27

21. Elezi S, Kastrati A, Hadamitzky M, et al. Clinical and angiographic follow-up after balloon angioplasty with provisional stenting for coronary in-stent restenosis. Catheter Cardiovasc Interv 1999;48:151-56

22. Levy EI, Turk AS, Albuquerque FC, et al. Wingspan in-stent restenosis and thrombosis: incidence, clinical presentation, and management. Neurosurgery 2007;61:644-50

23. Yu W, Smith WS, Singh V, et al. Long-term outcome of endovascular stenting for symptomatic basila artery stenosis. Neurology 2005;64:1055-57

24. Fiorella DJ, Levy EI, Turk AS, et al. Target lesion revascularization after Wingspan: assessment of safety and durability. Stroke 2009;40:106-10

25. Turk AS, Levy EI, Albuquerque FC, et al. Influence of patient age and stenosis location on Wingspan in-stent restenosis. AJNR Am J Neuroradiol 2008;29:23-27

26. Cosottini M, Michelassi MC, Bencivelli W, et al. In stent restenosis predictors after carotid artery stenting. Stroke Res Treat 2010;14:2010

27. Zhu SG, Zhu YL, Zhou ZM, et al. Effect of lesion length on in-stent restenosis after intracranial stenting. Zhonghua Yi Xue Za Zhi 2010;90:2040-43

28. Chen HY, Hermiller J, Sinha AK, et al. Effects of stent sizing on endothelial and vessel wall stress: potential mechanisms for in-stent restenosis. J Appl Physiol 2009; 106:1686-91 